Théologiques

Théologiques

\title{
Figures du discernement et construction identitaire à Qumrân
}

\section{Jean Duhaime}

Volume 22, numéro 2, 2014

Le discernement spirituel

URI : https://id.erudit.org/iderudit/1035685ar

DOI : https://doi.org/10.7202/1035685ar

Aller au sommaire du numéro

\section{Éditeur(s)}

Faculté de théologie et de sciences des religions, Université de Montréal

\section{ISSN}

1188-7109 (imprimé)

1492-1413 (numérique)

Découvrir la revue

\section{Citer cet article}

Duhaime, J. (2014). Figures du discernement et construction identitaire à Qumrân. Théologiques, 22(2), 17-49. https://doi.org/10.7202/1035685ar

\section{Résumé de l'article}

Les manuscrits de la mer Morte témoignent d'une communauté qui affirme connaître le « véritable " plan de Dieu sur le monde et y répondre de manière appropriée en pratiquant un style de vie rigoureux, sous la conduite de maîtres éclairés. Pour soutenir cette prétention, elle s'appuie sur deux figures principales de discernement, le " maître de justice " (moreh tsèdeq), dont l'enseignement a joué un rôle déterminant dans la structuration de la communauté, et le "sage » ou l'« instructeur " (maśkîl), chargé de montrer la voie aux recrues et de faire progresser les membres. Dans cet article, chacune de ces figures-types est d'abord présentée à partir des textes qui la mentionnent, après quoi on identifie la forme que prend le discernement qui lui est attribué et comment cette représentation contribue à la construction et au renforcement de l'identité sociale du groupe. 


\title{
Figures du discernement et construction identitaire à Qumrân
}

\author{
Jean Duhaime* \\ Théologie et sciences des religions \\ Université de Montréal (Canada)
}

Au tournant de notre ère, une communauté juive dissidente vivant à Qumrân, au bord de la mer Morte, a préservé dans sa «bibliothèque » des écrits qui révèlent sa prétention à connaître le "véritable " plan de Dieu sur le monde et à y répondre de manière appropriée en pratiquant un style de vie rigoureux, sous la conduite de maîtres éclairés ${ }^{1}$. Pour soutenir une telle prétention, la communauté devait s'appuyer sur des figures crédibles auxquelles ce discernement était attribué et que les disciples étaient invités à suivre. Deux figures-types principales émergent des documents communautaires: celle du «maître de justice» (moreh tsèdeq), dont l'enseignement a joué un rôle déterminant dans la structuration de la communauté, et celle du sage ou de l' "instructeur» (maśkîl), chargé de montrer la voie aux recrues et de faire progresser les membres. Comme contribution à ce dossier sur le discernement, je propose d'explorer ces deux figures-types

* Jean Duhaime est professeur émérite de la Faculté de théologie et des sciences des religions de l'Université de Montréal. Spécialiste de la Bible hébraïque et du judaïsme ancien, particulièrement des manuscrits de la mer Morte, il a publié récemment une traduction commentée de la Règle de la guerre (2013) "War Scroll», dans L. Feldman, J. Kugel et L. Schiffman, dir., Outside the Bible: Ancient Jewish Writings Related to Scripture, Philadelphia, Jewish Publication Society, p. 3116-3151. Il a aussi codirigé avec Peter W. Flint un collectif d'études sur le sujet: (2014) Célébrer les manuscrits de la mer Morte. Une perspective canadienne, Montréal/Paris, Médiaspaul.

1. Sur la bibliothèque de Qumrân, voir Duhaime et Legrand (2010). Les textes de Qumrân cités sont généralement traduits sur les originaux d'après l'édition officielle qu'on trouve dans la collection «Discoveries in the Judaean Desert» (DJD 19552010), le Dead Sea Scrolls Reader (Parry et Tov 2004) et l'édition électronique publiée par Tov (2006). Dans toutes les traductions et citations de notre article, des crochets signalent soit une lacune [...], soit une reconstruction [xxx]. Les parenthèses indiquent une omission (...) ou un ajout $(\mathrm{xxx})$.

(C) Revue Théologiques 2014. Tout droit réservé. 
complémentaires qui ont participé à l'élaboration, au maintien et à la consolidation de l'identité sectaire du ou des groupes qu'ils représentent et à la socialisation de ses membres. Chacune sera d'abord présentée à partir des textes qui la mentionnent, après quoi nous chercherons à identifier la forme que prend le discernement qui lui est attribué et comment cette représentation contribue à la construction de l'identité sociale du groupe qui s'en réclame.

\section{Le Maître de justice}

\subsection{Le personnage et les textes}

Le titre de "Maître de justice" (moreh tsèdeq) désigne un personnage auquel quelques textes de Qumrân reconnaissent un rôle important dans l'histoire des origines du mouvement de réforme dont ils sont issus ${ }^{2}$. On retrouve cette figure de manière explicite une vingtaine de fois, principalement dans le Document de Damas (CD), le Commentaire (pècher) d'Habaquq (1QpHab), celui de Michée (1Q14) et deux commentaires de psaumes (4Q171; 4Q173)33. Le Maître de justice est aussi considéré par plusieurs, avec une certaine vraisemblance, comme l'auteur ou le protagoniste d'autres écrits trouvés à Qumrân, notamment de la lettre halakhique (4QMMT) et de certains hymnes à caractère autobiographique $\left(1 \mathrm{QH}^{\mathrm{a}} \mathrm{X}-\mathrm{XVII}\right.$; voir Knibb 2000). Sans lever cette ambiguïté, ce qui nécessiterait une étude approfondie de la question, nous examinerons l'ensemble de ces textes.

\section{La Lettre halakhique}

La «Lettre halakhique» (4QMMT) est un texte reconstitué par Qimron et Strugnell à partir de manuscrits fragmentaires ${ }^{4}$. Elle se présente comme le

2. Sur le Maître de justice, voir Brooke (2005); Finsterbusch (2013); Harkins (2012); Jokiranta (2006; 2008); Knibb (2000); Laperrousaz (1997); Newsom (2004); Stuckenbruck (2010); Ulfgard (1998).

3. Voir surtout CD I 11; XIX 35 - XX 1; XX 28.32; 1QpHab I 13; II 2; V 10; VII 4; VIII 3; IX 9; XI 5; 1Q14 8-10 6; 4Q171 1-10 III 15.19; IV 8.27; 4Q173 1 4; 22. Peut-être parce qu'elle appartient au passé, la figure du Maître de justice est absente d'écrits davantages préoccupés par le présent et l'avenir, comme la Règle de la Communauté (1QS; 4Q255-264a), la Règle de la congrégation (1QSa), le Recueil de bénédictions (1QSb) et la Règle de la guerre (1QM; 4Q491-497; voir 4Q285; 471; 11Q14).

4. Qimron et Strugnell $(1994,43-63)$ : ce texte est un assemblage de six manuscrits fragmentaires se recoupant partiellement (4Q394-399 ou 4QMMT ${ }^{\text {a-f }}$, présentés aux 
plaidoyer envoyé par les responsables d'un groupe («nous») à un dirigeant d'Israël, probablement un des souverains maccabéens, et à son peuple. Ils lui expliquent leur manière de comprendre certaines prescriptions de la Torah (la Loi de Moïse) et de les appliquer. Leurs opinions, généralement très strictes, se démarquent de celles d'un groupe rival qu'ils désignent comme «eux». Ils espèrent gagner ce dirigeant à leur cause et l'enjoignent d'adopter leur point de vue, dans l'intérêt de la nation.

Les auteurs de ce texte ne revendiquent aucune «révélation» ou inspiration particulière. Ils présentent leur exposé comme "quelques-unes de nos paroles" (ou "règles ", devarim) concernant des "actions" prescrites par la Loi (4QMMT B 1-2). Dans certains cas, l'énoncé de l'objet du litige est simplement suivi d'une brève description de la pratique des "autres" qu'on dénonce comme contraire à la Loi :

[À propos de l'offrande végétale pour le sacrifice] de paix dont ils laissent les restes d'un jour subsister jusqu'au lendemain: [il est] pourtant [écrit] que l'offrande végétale [doit être consommée] après les graisses et la viande, le jour de leur sacrifice. (4QMMT B 9-11; voir Lv 6,21).

Ailleurs, les expressions "nous disons que », "nous pensons que » introduisent un énoncé sur la manière dont le point débattu doit être compris, vraisemblablement à partir d'une prescription apparentée: «Et à propos de [l'impureté des restes (?)] humains: nous disons que tout ossement, qu'il soit [décharné (?)] ou complet, doit être traité selon la prescription applicable à la personne morte (naturellement) ou tuée » (4QMMT B 72-74; voir $\mathrm{Nb}$ 19,16-18).

Dans la conclusion de la lettre, les auteurs affirment s'être séparés du reste du peuple à cause de leur manière de comprendre et d'observer la Loi et ils proclament leur intégrité (4QMMT C 7-9). Ils font directement appel à leur destinataire chez qui ils disent avoir décelé les qualités requises pour comprendre la Loi : ils l'invitent à évaluer leur point de vue et à s'y rallier:

(...) nous avons constaté que tu as de la perspicacité et une connaissance de la Loi. Réfléchis à toutes ces questions et demande-Lui de redresser ton conseil et d'éloigner de toi les mauvais desseins et le conseil de Bélial de sorte que tu te réjouisses, dans l'avenir, d'avoir trouvé correctes quelques-unes de nos déclarations. Cela te sera compté comme justice, de faire ce qui est droit et juste devant Lui, pour ton bien et celui d'Israël. (4QMMT C 26b-32)

p. 3-42). On y réfère par section (A, B, C) et par ligne. Voir aussi Berthelot (2013; nouvelle édition du texte hébreu et traduction annotée intégrant les débats récents). 
Bien que le "Maître de justice» ne soit jamais mentionné directement dans la Lettre halakhique, les éditeurs estiment vraisemblable qu'elle ait été expédiée par celui-ci et d'autres responsables de sa communauté à un grand prêtre qui aurait été considéré comme «impie» seulement après avoir rejeté cet appel (Qimron et Strugnell 1994, 114-121). Même si cette attribution n'est pas assurée (voir Berthelot 2013, 647-648), la lettre remonte probablement aux origines du mouvement dans lequel le Maître s'est inséré et au sein duquel il a exercé une action située dans la même ligne de fond et se réclamant des mêmes principes.

\section{Le Document de Damas}

Le début du Document de Damas raconte les origines d'une communauté de «la nouvelle alliance» en la présentant comme «une racine de plantation » suscitée par le Dieu d'Israël et d'Aaron quelque 390 ans après qu'il eût livré son peuple coupable aux mains de Nabuchodonosor, une référence à la chute de Jérusalem au vi ${ }^{\mathrm{e}}$ s. a.n.è. (CD I 3-7; voir Éz 4,4-5). Reconnaissant leur culpabilité, ces repentis qui «cherchaient Dieu d'un cœur parfait ", auraient tâtonné pendant une vingtaine d'années, jusqu'à ce que Dieu «leur suscite un maître de justice pour les conduire dans la voie de son cœur»(I 11a). La suite du texte évoque, par opposition, le châtiment réservé à une "congrégation de traîtres ", égarés par un railleur qui répand le mensonge et attire sur eux les «malédictions de l'alliance » (I $11 \mathrm{~b}-$ II 1).

La figure du maître apparaît encore dans une exhortation où il est question du bonheur promis aux fidèles. Ceux qui «tendent l'oreille à la voix du Maître de justice et ne rejettent pas les décrets de justice quand ils les entendent » sont promis au salut: "Eux se réjouiront et seront en liesse, leur cœur sera fort et ils l'emporteront sur les fils du monde, Dieu leur pardonnera et ils verront son salut car ils se sont réfugiés dans son saint nom » (XX 31-32). Par contre, quiconque est «entré dans l'alliance» mais aura «transgressé la limite de la Loi », sera «retranché du camp» lorsque la gloire de Dieu se manifestera à Israël (XX 25-26). Le Maître est vraisemblablement perçu comme celui qui, par son interprétation et son enseignement, a contribué à préciser cette limite.

\section{Les commentaires bibliques}

Les commentaires bibliques dans lesquels on mentionne le Maître de justice sont assez fragmentaires, à l'exception de celui d'Habaquq. Dans les 
fragments 8-10 du commentaire de Michée, il est opposé à un «baveur » de mensonges qui égare les simples; c'est vraisemblablement quelqu'un qui guide «tous ceux qui s'offrent pour être agrégés aux élus [de Dieu]» et qui «seront sauvés du jour [du jugement...]» (1Q14 frag. 8-10 1-9).

Le commentateur du livre d'Habaquq lit ce texte prophétique comme une prédiction, faite plusieurs siècles à l'avance, concernant des événements qui se sont déroulés dans sa communauté. Le Maître de justice y est assimilé au "juste " traqué par l'impie, un opposant dont l'identité est perdue (1QpHab I 12-13; voir Ha 1,4). Peu après, dans un commentaire d'Ha 1,5, le Maître est présenté comme un porte-parole de Dieu ayant affronté l'incrédulité de traîtres qui ont préféré suivre un "homme de mensonge » (II 1-3). Dans le même contexte, il est également fait référence aux "[traî]tres des jours à venir ", identifiés comme «des ennemis de l'alliance » qui ne croiront pas les propos du «prêtre auquel Dieu a donné l'intelligence pour expliquer toutes les paroles de ses serviteurs les prophètes par lesquels Dieu a annoncé tout ce qui allait arriver à son peuple et à sa communauté » (II 6-10). Le contexte suggère qu'il pourrait s'agir aussi du Maître de justice, dont les enseignements seraient transmis aux générations futures (Stuckenbruck 2010, 20-30). Un autre passage laisse entendre que la dispute entre le Maître et l' "homme de mensonge " portait sur l'interprétation de la Loi, que ce dernier est accusé d'avoir rejetée (V 9-12).

Dans son interprétation d'Ha 2,1-2, le commentateur explique qu'en demandant à Habaquq d' "écrire la vision ", Dieu lui faisait consigner "tout ce qui adviendrait à la génération à venir ", sans lui révéler le moment où cela se réaliserait (VII 1-2). C'est seulement au Maître de justice, plusieurs siècles plus tard, que Dieu aurait «fait connaître tous les mystères des paroles de ses serviteurs les prophètes ", le rendant ainsi capable d'en dévoiler le sens à ses disciples (VII 3-5).

Le texte d'Ha 2,4b «le juste vivra à cause de sa fidélité » est aussi compris en fonction du Maître: en effet, "tous ceux qui observent la Loi dans la maison de Juda» et qui font preuve de «fidélité envers le Maître de justice ", Dieu les "délivrera de la maison du jugement » où périront sans doute les impies (VII 1-3).

Le commentaire d'Habaquq évoque encore un crime perpétré par le «Prêtre impie» contre le «Maître de justice et les hommes de son conseil » (IX 9-10) et une agression qu'il aurait commise contre lui en l'attaquant dans sa «maison d'exil» un jour de Kippour» (XI 2-8), que la communauté du Maître observait selon son calendrier propre. 
Le Maître est mentionné, enfin, dans deux commentaires de psaumes (4Q171 et 173). Le second est trop fragmentaire pour livrer une information utile. Le premier comporte une interprétation du Psaume 37. Le maître y est identifié au juste qui bénéficie de la protection divine:

Car du SEIGNEUR [(sont) les pas de l'homme. Ils] sont affermis et son chemin lui plaît. S'il [trébu]che, il n'est [pas] terrassé car le SEI[GNEUR lui tient la main]. Son interprétation (concerne) le "prêtre", le maître de [justice que] Dieu a choisi comme pilier. Car il l'a affermi pour lui construire une congrégation de [... et] son [che]min va droit à la vérité. (4Q171 frag. 1-10 III 14-17; voir Ps 37,23-24)

Les versets 32-33 du Psaume 37 sont lus comme une annonce de l'embuscade que le Prêtre impie aurait tendue au Maître de justice, cherchant à le faire mourir, peut-être à propos de "la loi qu'il lui a envoyée ". Le commentateur y lit la promesse que Dieu n'abandonnerait pas le Maître aux mains de son adversaire et qu'il châtierait ce dernier (4Q171 frag. 1-10 IV 7-10).

\section{Les Hymnes}

Le rouleau des Hymnes $\left(1 \mathrm{QH}^{\mathrm{a}}\right)$ comporte un groupe de textes dans lesquels un dirigeant de la communauté affirme être en possession d'un savoir communiqué par Dieu pour guider sa communauté dans la vérité et la conduire au salut (X 5 - XVII 36; Stegemann et al. 2009). Gert Jeremias (1963) croyait y reconnaître le Maître de justice et être en mesure d'établir une corrélation entre les descriptions des Hymnes et les références au Maître de justice dans les autres textes qumraniens. Cette hypothèse, largement suivie, est commandée surtout par un intérêt historique. Quelques chercheurs contemporains sont toutefois réticents à l'endosser et s'intéressent davantage à la performance rhétorique de ces compositions poétiques (voir Harkins 2012; 2013; Newsom 2004). Bien que l'expérience que ces hymnes décrivent puisse effectivement correspondre à celle du Maître de justice, elle conviendrait tout autant à la figure-type du dirigeant communautaire, envers lequel on chercherait à renforcer l'attachement des membres (voir Newsom 2004, 289-346). Quoi qu'il en soit, il est pertinent pour notre propos d'explorer le discernement qui lui est attribué.

Le locuteur du premier hymne de cette section (X 5 - 21) se présente comme quelqu'un dont Dieu a fait "une fondation de vérité et d'intelligence pour ceux dont la voie est droite » (X 12), "un interprète expert de mystères merveilleux» (X 15), «un homme dans la bouche duquel tu as 
établi l'instruction et dans le cœur (duquel) tu as mis l'intelligence pour ouvrir la source de la connaissance à tous les intelligents» (X 19-20).

Le septième hymne déploie une thématique du même genre (XII 6 XIII 6). S'adressant à Dieu, un personnage similaire lui rend grâce car, dit-il, "tu as illuminé ma face pour ton alliance» (XII 6). Il se décrit comme un être auquel Dieu a fait comprendre ses «mystères merveilleux" et en qui il a manifesté sa force (XII 28-29). Il affirme être écouté par "ceux qui marchent dans la voie de ton cœur» (XII 25), de nombreux disciples dont Dieu a aussi «illuminé la face» grâce à lui (XII 28). Il a pour adversaires des interprètes de mensonges qui cherchent Dieu «d'un cœur double» et ne sont pas fidèles à sa vérité (XII 15), se laissent leurrer par de faux prophètes, refusent la parole de Dieu et rejettent «la vision de connaissance» (XII 17-19). C'est pourquoi ils ont égaré son peuple (XII 8) et «ont retiré le breuvage de la connaissance aux assoiffés » pour les abreuver de vinaigre (XII 12).

Dans le neuvième hymne (XIII 22 - XV 8), un dirigeant évoque la jalousie et la trahison dont il a été victime de la part de "ceux qui sont attachés à mon conseil » (XIII 25-26): ils auraient répandu auprès des «fils de destruction" des calomnies à propos du «secret que tu as caché en moi »(XIII 27). Le même personnage se plaint que ceux qui s'étaient «attachés à mon témoignage se sont laissés persuader par des interprè [tes égarés]» (XIV 22).

\subsection{Quelle forme de discernement?}

Quelle forme de discernement peut-on dégager de ces textes? Les auteurs de la lettre halakhique légitiment leur point de vue en se présentant comme des gens intègres, chez qui ne se trouve "ni tromperie, ni mensonge, ni mal» (4QMMT C 7-9). Dans l'ensemble des textes qui évoquent explicitement le Maître de justice, celui-ci apparaît comme un juste, vraisemblablement un prêtre bénéficiant de l'appui de Dieu pour devenir le guide des fidèles (CD I 11; XX 31-32; 1Q14 frag. 8-10 1-9; 1QpHab I 12-13; 4Q171 frag. 1-10 III 14-17). Dans les Hymnes, le protagoniste se définit comme quelqu'un qui «cherche Dieu» et dont la face a été «illuminée» par lui $\left(1 \mathrm{QH}^{\mathrm{a}} \mathrm{XII} 6\right)$.

L'enjeu principal de la lettre halakhique est la compréhension des prescriptions de la Torah. En opposant leur point de vue à celui de leurs adversaires, les auteurs de la lettre revendiquent être les détenteurs du savoir «correct» concernant la Loi (4QMMT C 30). Le discernement 
attribué au Maître de justice concerne également l'interprétation adéquate de la Loi, dont il sait préciser les limites (CD XX 25-26; 1QpHab V 9-12; voir 4Q171 f. 1-10 IV 7-10). De plus, Dieu l'a gratifié d'un talent particulier pour comprendre et expliquer les paroles des prophètes dont il lui a révélé les mystères (1QpHab II 6-10; VII 3-5). Les Hymnes semblent faire écho à ces deux aspects du discernement en évoquant un dirigeant qui est "une fondation de vérité et d'intelligence pour ceux dont la voie est droite ", quelqu'un qui comprend «les mystères merveilleux" et en qui Dieu a caché «son secret» (1QH $\mathrm{QH}^{\mathrm{a}}$ X 12.15 ; XII 28-29; XIII 27).

Les auteurs de la lettre halakhique accomplissent une sorte de mission auprès du leader de la nation en lui proposant ce qu'ils considèrent comme une voie de justice, pour son bien et celui d'Israël... (4QMMT C 26-32). La perspicacité exceptionnelle du Maître de justice apparaît comme un don d'origine divine en fonction de la mission qui lui est confiée, celle de montrer à sa communauté la voie conduisant au salut. La fidélité à l'égard du Maître devient alors une condition essentielle pour y accéder (CD XX 31-32; 1Q14 frag. 8-10 1-9; 1QpHab VII 1-3; 4Q 171 frag. 1-10 III 14-17). Selon les Hymnes, le Maître ou un dirigeant du même genre est écouté par ceux qui sont accordés au cœur de Dieu et dont la face est illuminée par son intermédiaire (1QH $\left.\mathrm{Q}^{\mathrm{a}} \mathrm{XII} 25.28\right)$.

Ce rôle de guide s'exerce toutefois dans un contexte polémique. La lettre halakhique fait état d'une compétition entre deux groupes («nous» et "eux») autour de l'interprétation de la Loi et évoque des "plans mauvais» qu'il faut écarter (4QMMT C 29). Dans les commentaires bibliques, le Maître est confronté à des ennemis de l'extérieur, en particulier au Prêtre impie qui le traque et cherche à le faire périr (1QpHab I 12-13; IX 9-10; XI 2-8). Il se heurte aussi à un "baveur de mensonge", à un "railleur " qui égare une congrégation de traîtres, etc. (1QpHab II 1-3.6-10). Ce même contexte se retrouve dans les Hymnes où le protagoniste principal est confronté à des interprètes de mensonge qui égarent le peuple de Dieu et même à "ceux qui s'étaient attachés à mon témoignage » ... (1QH $\mathrm{H}^{\mathrm{a}} \mathrm{XII}$ 12.15.17-19; XIII 25-26; XIV 22).

\subsection{Quelle contribution à la construction identitaire?}

Aucun des textes qui parlent explicitement du Maître de justice ou qui en évoquent éventuellement la figure n'a le souci d'établir clairement les fondements du discernement qui lui est attribué. Le statut d'élu de Dieu et les qualités exceptionnelles du Maître semblent s'être imposés à ses disciples, 
comme une évidence, à travers le rôle déterminant qu'il a joué dans la structuration de la communauté, la fécondité des orientations qu'il lui a données et sa capacité exemplaire de résister face aux attaques des ennemis et aux calomnies des dissidents. Il est ainsi devenu une figure de référence dont la personne et l'action ont inspiré non seulement ses contemporains, mais également leurs successeurs.

La lettre halakhique a survécu en six exemplaires et a été transmise d'une génération à l'autre pendant plusieurs décennies. Ses orientations légales sont semblables à celles du Document de Damas et d'autres textes trouvés à Qumrân. Sur le plan historique, ce document proposait aux membres d'un groupe marginal une justification pour sa rupture avec le Temple de Jérusalem et ses dirigeants: il s'agit d'un choix auquel "nous» avons été contraints, face aux pratiques illégitimes des autorités en place, par fidélité à notre compréhension de la Loi. Mais le document a sans doute aussi joué un rôle dans la formation d'une tradition d'interprétation de la Loi: en se penchant sur les cas exemplaires exposés dans cet écrit, les membres ou dirigeants successifs de ce groupe apprenaient de leurs prédécesseurs les principes dont ils s'étaient inspirés, afin de les assimiler et d'en tirer des règles applicables à d'autres cas semblables.

Le souvenir du Maître de justice, dans le Document de Damas et surtout les commentaires bibliques, est du même ordre: à la fois historique et typologique. Håkan Ulfgard note le rôle normatif qu'on lui attribue en raison de son inspiration divine et de sa fonction d'interprète de la Loi en contexte eschatologique:

It was his halakah that was to be followed by God's chosen people in the New Covenant, just as it was he who was to disclose God's hidden secrets in the words of the prophets, showing how these could be applied to the community of the elect at the End of Days. (Ulfgard 1998, 332)

Jutta Jokiranta attire l'attention sur le caractère typologique de la description du Maître dans les commentaires bibliques: sa situation d'élu, bénéficiant d'une révélation en vue d'une mission particulière mais persécuté par des ennemis et des traîtres, s'apparente étroitement à celle du groupe lui-même, dont il constitue le prototype:

He represents the in-group and stands opposite to the out-groups. This prototypical image serves a group identity, in which belonging to the group is necessary to gain access to God's covenant, and afflictions are one part of testing the righteous. This kind of group identity could better survive the threats posed by the apostates, by their own minority position, or by political disturbances ». (Jokiranta 2006, 263) 
Sa figure historique sert donc la mémoire du groupe en lui fournissant un lien avec son passé, une représentation de son fondement et de son identité, ainsi qu'une norme d'évaluation de la fidélité de ses membres: "Collective memory of the leader intensifies the in-group members' identification with the group, providing the necessary continuity with its past » (Jokiranta 2008, 96).

L'étude de Loren T. Stuckenbruck va dans le même sens. L'activité du Maître n'est pas confinée au passé. Elle devient une véritable clé herméneutique, notamment pour l'auteur du commentaire d'Habaquq:

For the writer of the pesher, the Teacher's interpretation of the prophets are not simply remote activities that reinforce the uniqueness of the Teacher: instead, the author finds in the Teacher's authority a hermeneutical key that opens up, in principle, the way for him (and therefore his community) to discover afresh the meaning of the text for circumstances of his own days. (...) the author takes the mantel of the Teacher's authority upon himself, by providing a running commentary on Habakkuk with contemporary events in view» (Stuckenbruck 2010, 33).

La mémoire du Maître était donc conservée par son imitation plutôt que par la préservation des détails de sa biographie ou de son enseignement, somme toute assez peu nombreux dans le Document de Damas et dans les commentaires bibliques: "For the community, the appropriate memory of him is through the mimesis of the authority that he claimed and the praxis that he initiated" (Stuckenbruck 2010, 36). La même mimesis joue également à propos de sa biographie. Son nom, connu probablement des initiés, n'est pas conservé dans ces textes où l'on évoque plutôt des événements qui ont valeur de modèle pour le groupe: "Certain events from the Teacher's life were chosen to be preserved because they were closely bound up with the community's self-understanding and activity » (Stuckenbruck 2010, 48).

Carol A. Newsom observe un phénomène semblable en ce qui concerne les Hymnes attribués au Maître de justice ou à un dirigeant apparenté. Selon elle, il est possible, mais finalement indémontrable, que ces Hymnes aient été composés par le Maître de justice. Mais leur valeur est ailleurs. Ils incarnent en quelque sorte le «mythe du leadership » de la communauté et ont servi, au fil de leur lecture et de leur récitation à façonner l'identité de la communauté et à proposer des réponses aux questions inhérentes à la vie d'un groupe de type sectaire (Newsom 2004, 288-289). En évoquant la délimitation des frontières entre les membres du groupe et les gens de l'extérieur, la confrontation à des dissensions internes, le rôle d'un leader 
dans la construction de sa collectivité, ils explicitent à leur façon les principales responsabilités qu'assume ordinairement un chef de communauté: «(...) in their repetition over decades of use, they would have come to form the dramatized expression of the habitual fonctions of leadership within the sectarian community" (Newsom 2004, 298). Présentés dans un langage fortement teinté d'émotion, ils auraient servi à renforcer la loyauté envers le chef de la communauté et à faire face à l'opposition et à la défection:

Whether the compositions were understood to represent the persona of the Teacher or of the leader who recited them, they serve above all to construct a figure who is a compelling object of loyalty. Who he is in relation to God, the community, and their opponents is at the heart of these self-presentations. (...) These compositions build up the community in other ways as well, articulating important aspects of its figured world. (...) They give positive meaning to the rejection of (or indifference to) the sect by other Jews and provide an interpretive narrative context in which the truth possessed by the sect is validated. (Newsom 2004, 345)

\section{Le maśkîl}

\subsection{Le personnage et les textes}

Le terme maśkîl se trouve une trentaine de fois dans la Bible hébraïque: il s'agit d'une forme participiale du verbe śākal (hiphil) qui évoque l'intelligence ou la sagesse d'une personne, sa conduite avisée ou sa réussite (Elgvin 2013; Koenen 1993). Il sert à décrire le succès de David, le bon sens de celui qui récolte au moment opportun ou qui sait mesurer son langage, etc. (1 S 18,14-15; Pr 10,5.19). Il est employé comme terme technique dans plusieurs titres de psaumes "d'instruction" (Ps 32,1; 42,1; etc.). Le pluriel maśkîlim désigne soit des Lévites (2 Ch 18,22), soit des sages qui, après avoir guidé la multitude et avoir péri au temps de l'angoisse, s'éveilleront pour resplendir à tout jamais (Dn 11,33-35; 12,3.10). Dans les textes de Qumrân, maśkîl apparaît comme substantif une quarantaine de fois ${ }^{5}$. On considère généralement qu'il désigne l'un des principaux

5. CD XII 21; XIII 22; 1QS III 13; IX 12.21; 1Q28b I 1; III 22; V 20; 1QHa V 12; VII 21; XX 7.14; XXV 34; 8 10; 4Q256 IX 1; 4Q258 I 1; VIII 5; 4Q259 III 7; IV 2; 4Q266 5 i 17; 9 iii 15; 4Q298 1-2 i 1; 4Q382 14 1; 4Q400 3 ii + 5 8; 4Q401 1-2 1; 4Q403 i 30; ii 18; 4Q405 20 ii + 21-22 6; 4Q406 1 4; 4Q417 1 i 25; 4Q418 $81+81 \mathrm{a} 17 ; 238$ 1; 4Q418a 19 2; 4Q421 1a ii + 1b 10.12; 4Q427 8 ii 10.17; 12 ii 3; 4Q433 2 2; 4Q461 1 6; 4Q510 1 4; 2 i 1; 11Q17 VII 9. Voir Abegg et al. (2003, 489); Tov (2006, ad loc.). 
responsables du groupe, un homme instruit, éclairé et réfléchi, qui agit comme maître et guide tant auprès des novices que du reste de sa communauté6. Cette figure apparaît dans des règles (Document de Damas et Règle de la communauté), des écrits de sagesse (Instruction, Paroles du maśkîl aux fils de l'aurore, Voies de la justice) et des recueils poétiques ou liturgiques (Recueil de bénédictions, Hymnes, Chants pour l'holocauste du sabbat, Chants pour le maśkîl).

\subsection{Règles}

\section{Document de Damas}

Le Document de Damas ne mentionne le maśkîl qu'à deux reprises, dans des rubriques. La première annonce une série de prescriptions qui lui sont destinées: "Voici les décrets pour le maśkîl, selon lesquels il se conduira avec tout vivant, selon la prescription propre à chaque temps » (CD XII 21 ; par. 4Q266 frag. 9 ii 7). Mais la liste semble avoir été omise, puisque cette rubrique est suivie immédiatement d'une "règle pour l'organisation des camps» (CD XII 22-23) ${ }^{7}$. La seconde occurrence se trouve dans une portion abimée du texte (CD XIII 22; par. 4Q266 frag. 9 iii 15; voir 4Q266 frag. 5 i 17).

Règle de la communauté

La figure du maśkîl apparaît à plusieurs reprises dans quelques exemplaires de la Règle de la communauté. Dans celui de la grotte 1, on a proposé avec une certaine vraisemblance de restaurer ce terme au tout début (1QS I 1; voir Guilbert 1961, 21) ${ }^{8}$, dans le titre de la section qui énonce les objectifs du groupe (I 1-15), décrit le rituel d'entrée dans l'alliance et celui de son renouvellement annuel (I 16 - II 25) et traite le cas du «faux converti » qui refuse de se soumettre humblement à la discipline (II 25 - III 12).

6. Sur le maśkîl à Qumrân, voir principalement les divers points de vue de Angel (2012); Elgvin (2013); Hawley (2006); Hempel (2006); Lange (2008); Newsom (1990a; 1990b; 2004).

7. Une rubrique similaire se trouve dans la Règle de la communauté (1QS IX 12, ciaprès), au début d'une liste de préceptes; selon Cothenet (1963, 199), l'auteur ou compilateur du Document de Damas pourrait y référer en reproduisant son introduction. Sur cette question, voir Hempel (1998, 105-106, 114-121, 189).

8. Il en est de même pour la Règle de la guerre (1QM), qui ne mentionne le maśkîl nulle part ailleurs, toutefois. 
On rencontre la première occurrence sûre dans l'entête de l'Instruction sur les deux esprits (1QS III 13 - IV 26): "C'est au maśkîl de faire comprendre et d'enseigner à tous les fils de lumière la nature de tous les fils d'homme, selon toutes leurs sortes d'esprits et les caractéristiques de leurs œuvres en leurs générations, et selon la visite où ils seront affligés ainsi que les temps de leur bonheur»(III 13-15a). Ce texte attribue au «Dieu des connaissances" la création, selon un plan préétabli, de deux esprits antagonistes associés respectivement à la lumière et aux ténèbres ou, sur le plan moral, à la vérité et à la perversité (III 15b - IV 1); il énonce ensuite "les conseils » de l'esprit pour les fils de vérité dans le monde et les récompenses eschatologiques auxquelles ils conduisent (IV 2-8) et, par opposition, ceux de l'esprit de perversité et les châtiments qui en résultent (IV 9-13); la dernière section évoque une lutte constante des esprits dans le cœur de chacun, jusqu'à la purification eschatologique des élus (IV 15-26):

(...) Dieu, dans son intelligence mystérieuse et sa sagesse glorieuse, a prévu un terme à l'existence de la perversité. Au moment de la visite, il l'anéantira à jamais. (...) Alors Dieu purifiera par sa vérité toutes les œuvres de l'humain, et s'affinera des fils d'homme, détruisant totalement tout esprit de perversité de leurs membres charnels, les purifiant par l'Esprit Saint de toute activité méchante. Il fera jaillir sur lui l'Esprit de Vérité, comme de l'eau lustrale, (pour le nettoyer) de toutes les abominations mensongères et de la pollution par un esprit de souillure, pour instruire les (hommes) droits de la connaissance du Très-Haut et pour faire comprendre la sagesse des fils du ciel à ceux dont la voie est parfaite. Car Dieu les a choisis pour une alliance éternelle et toute la gloire d'Adam leur appartiendra, sans perversité. (1QS IV 18-23)

Les sections suivantes comportent plusieurs séries de prescriptions concernant le déroulement de la vie communautaire (1QS V 1 - IX 11). Dans l'exemplaire de la grotte 1, la première est présentée comme «la règle pour les hommes de la communauté, qui sont volontaires pour se convertir de tout mal et pour s'attacher à tout ce qu'il a commandé selon sa volonté» (1QS V 1). Toutefois, dans deux manuscrits de la grotte 4 correspondant à une autre recension de la Règle de la communauté, une version courte de cette section est qualifiée d' «interprétation pour le maśkîl à propos des hommes de la Torah qui sont volontaires pour se convertir de tout mal et pour s'attacher à tout ce qu'il a commandé » (4Q256 IX 1; 4Q258 I 1).

La dernière partie de la Règle porte spécifiquement sur les devoirs du maśkîl et illustre la manière dont il doit s'en acquitter (IX 12 - XI 22). Elle 
est introduite par une rubrique similaire à celle du Document de Damas: "Voici les décrets pour le maśkîl, selon lesquels il marchera avec tout vivant, selon la norme de chaque période et le poids de chaque homme» (IX 12). Il sera au fait de "tout ce qui a été révélé, de période en période» et apprendra "toute la compréhension qui a été trouvée selon les périodes et le décret du temps (de la fin)»(IX 13-14a). C'est également lui qui "séparera et pèsera les fils de justice ${ }^{9}$ selon leurs esprits ", et fera avancer chacun "à proportion de sa compréhension ", l'aimant ou le haïssant selon sa valeur (IX 14b-16a). S'abstenant de tout contact «avec les hommes de la fosse ", il ne discutera "la connaissance vraie et le juste jugement " qu'avec les élus, qu'il guidera dans la connaissance et la compréhension des mystères, "pour qu'ils marchent dans la perfection, chacun avec son prochain, en tout ce qui leur a été révélé »(IX 16b-21a). Sans coupure apparente, une nouvelle introduction mentionne encore le maśkîl et le discernement dont il doit faire preuve: «Voici les normes de conduite pour le maśkîl, en ces temps, concernant son amour et sa haine» (IX 21b; voir 4Q258 VIII 5 ; 4Q259 IV 2). Dans la brève section qui suit, on évoque effectivement la haine qu'il portera aux «hommes de la fosse », pour mieux faire ressortir le plaisir qu'il prendra à accomplir la volonté de Dieu, à se nourrir de sa parole et à chanter sa louange (IX 21c-26a).

Le reste du texte développe l'invitation à la louange. L'insistance est d'abord mise sur les moments appropriés pour s'y livrer, en synchronie avec «les temps qu'il a décrétés" et marqués dans les rythmes mêmes du cosmos (IX 26b - X 8). Vient ensuite un long hymne (X 9 - XI 22) dans lequel Dieu est notamment présenté comme une «fontaine de connaissance » qui fait connaître ses secrets merveilleux à ses fidèles et leur donne ainsi de s'unir aux fils du ciel:

Car, de sa fontaine de connaissance, il a fait jaillir sa lumière. Mon œil a perçu ses merveilles, et la lumière de mon cœur le mystère de ce qui sera et qui est, éternellement [... Il y a] en mon cœur la lumière de ses secrets merveilleux. Ce qui sera éternellement, mon œil [le] perçoit: la sagesse qui est cachée à l'humanité, la connaissance et la prudente discrétion [cachées] aux fils d'Adam, une fontaine de justice et un réservoir de puissance, ainsi qu'une source de gloire [cachés] à l'assemblée de la chair. Ceux que Dieu a choisis, il en a fait une possession éternelle. Il les a fait hériter du lot des saints. Avec les fils du ciel, il a uni leur assemblée, pour le Conseil de la Communauté. (XI 3-8)

9. Texte corrigé d'après 4Q259 III 10. 
Nourri de cette connaissance qui lui révèle la fragilité de la nature humaine en même temps que la grandeur de Dieu et de son dessein sur le monde, l'élu fait humblement confiance au créateur qui le soutient, le sauve, pardonne ses égarements et le purifie de toute souillure pour lui permettre de se tenir en sa présence à jamais (XI 9-17).

\section{2 Écrits de sagesse}

Instruction

L'«Instruction pour [le disciple] intelligent» (Mûsar le mévîn) est un ouvrage sapientiel probablement d'origine pré-essénienne représenté par au moins sept manuscrits (1Q26, 4Q415-418, 418a, 423; voir Strugnell et Harrington 1999). Il contient les conseils donnés à un disciple doué (mévîn) concernant l'argent, les relations sociales et la famille. On y trouve le substantif maśkîl à trois reprises. Mais le seul cas intéressant est un passage où, en conclusion d'un enseignement sur sa relation à Dieu, sur ses responsabilités et sur la manière dont il doit s'en acquitter, le disciple est invité à poursuivre son apprentissage en se mettant à l'écoute de ses maîtres: «[...] réfléchis beaucoup. Et par l'entremise de tes maśkîlim, augmente davantage ton acquis » (4Q418 frag. 81-81a 17; voir Pr 1,5; 9,9; 16,21).

Il est impossible de préciser si ce pluriel évoque un groupe de sages, comme dans le livre de Daniel, ou chacun des individus dont le disciple pourrait apprendre. Le savoir transmis par de tels maîtres s'enracine dans la notion de "mystère de l'existence ", centrale dans l'Instruction. Elle désigne la totalité de l'existence, passée, présente et à venir, "telle que Dieu l'a conçue depuis la création jusqu'à l'eschatologie»(Rey 2009, 292). Ayant leur fondement ultime en Dieu et dans sa vérité, l'univers et l'existence humaine obéissent à un plan bien arrêté, dont l'esprit humain attentif peut saisir l'essentiel. Correctement guidé dans son effort de réflexion et de méditation sur ce mystère, le disciple discernera le bien et le mal et saura ajuster sa conduite en vue du jugement divin:

[... Jour et nuit, médite sur le mystère de] l'existence. Scrute (le) continuellement. Alors tu connaîtras la vérité et la perversion, la sagesse [et la folie.] $\mathrm{Tu}$ [sauras quelle est] leur œuvre, en toutes leurs voies, ainsi que leur visite pour toute la durée du temps, et leur visite finale. Alors tu connaîtras (la différence) entre le bien et le mal [selon leurs] œuvres. Car le Dieu des connaissances est le fondement de la vérité. (C'est) par le mystère de l'existence (qu'il) l'a exposée. Ses œuvres, [il les a prévues en toute] sagesse; il l'a façonnée en toute habileté. Et la domination de ses œuvres (c'est) en tout [... 
qu'il l'a...] Il (l'a) exposée à leur intelligence en toute action, pour que (tous puissent) se conduire selon l'inclination de leur intelligence. Il a exposé pour [l’homme...] Dans l'abondance des (choses) intelligibles, sont révélés les secrets de son plan, ainsi que sa conduite parfaite [en toutes] ses œuvres. Recherche continuellement ces (réalités) et aie l'intelligence [de toutes] leurs conséquences. Alors tu connaîtras la gloire de [sa] for[ce ain]si que ses mystères merveilleux et ses puissantes œuvres. (4Q417 frag. 1 i 6-13a; voir 4Q418 frag. 43-45)

Paroles du maśkîl aux fils de l'aurore

4Q298 est un manuscrit de petite taille dont il subsiste huit fragments correspondant à deux colonnes (Pfann et Kister 1997). Il porte pour titre, en écriture hébraïque, "Paroles du maśkîl à tous les fils de l'aurore" (frag. 1-2 i 1). Le reste du texte recourt à un alphabet ésotérique ou cryptique, ce qui suggère un document réservé aux initiés. Les "fils de l'aurore", auxquels s'adressent ces propos sont probablement des «novices» qui émergent des ténèbres et sont en voie de devenir des «fils de lumière » au cours d'un processus d'insertion progressive dans le groupe des élus. Selon la Règle de la communauté, ce processus s'étendait sur deux ans (1QS VI 13-23).

Le texte commence par une invitation à l'écoute appuyée sur l'exemple de ceux qui ont déjà emprunté cette voie:

Prêtez-[moi] l'oreille, (vous) tous, hommes de cœur! [(Vous) qui poursui-] vez la justice, comprenez mes paroles! (Vous qui) recherchez la vérité, é[cou]tez mes paroles, toutes (celles qui) sortent de mes lèvres! [Ceux qui sa]vent ont scruté ces (choses) et les hom[mes (qui font?)] sa [vo]lonté se sont tournés vers le chemin de vie, et [(vers) la lumière (?)] éternelle [in-] sondable. (4Q298 frag. 1-2 i 1-4)

L'enseignement qui suit, dont le contenu préservé est très fragmentaire, porte sur l'ordre et les limites de l'univers créé (4Q298 frag. 1-2 ii 1 frag. 3-4 ii 3a). Dans un deuxième appel à l'écoute, le maître, s'adresse cette fois à «ceux qui savent»(4Q298 frag. 3-4 ii 4). Il pourrait s'agir de novices qui ont déjà acquis les notions de base, peut-être après une année d'enseignement, et sont maintenant invités à progresser dans la connaissance et la vie vertueuse:

Et maintenant prêtez l'oreille, [...]! Vous savez, écoutez! Hommes intelligents ac[croissez votre ac]quis! Vous recherchez la justice, (ajoutez) une 
conduite modeste! Vous con[naissez ...], ajoutez de la force! Hommes de vérité, recherchez [...]! Vous aimez la loyauté, ajoutez l'humilité! (4Q298 frag. 3-4 ii 3b-8a)

Cette exhortation introduisait vraisemblablement une nouvelle instruction, à caractère historique et eschatologique, où la perspective des temps à venir devait amener à reconsidérer le sens du passé: "Ajoutez la [con-] naissance des [jo]urs fixés, dont [je vais énon]cer l'interpré[ta]tion pour que vous compreniez la fin des temps et examiniez les événements d'au[tre-] fois pour comprendre [...]»(4Q298 frag. 3-4 ii 8b-10).

Voies de la justice

Le nom du maśkîl est mentionné au passage dans une section sapientiale d'un écrit intitulé "Voies de la justice ", un texte composite préservé en deux exemplaires qui combine des éléments d'organisation sectaire, des propos de sagesse et des règles concernant le temple (4Q420-421; Elgvin 1997; voir Kampen 2011, 284-299).

Dans la section qui nous intéresse, une admonition à se mettre sous «le joug de la sages[se]» (4Q421 frag. 1a ii + frag. 1b 10) sert d'introduction à une série d'énoncés décrivant le comportement qui devrait en résulter. Cela inclut notamment la capacité d'accepter les réprimandes du maśkîl, qui agit sans doute comme instructeur et guide dans l'apprentissage d'une sagesse rédemptrice:

[L'hom]me instruit et intelligent y puisera et [...L'homme ... sup]portera la réprimande du maśkîl. L’homme [...] pour marcher dans les voies de Dieu, pour accomplir la justice [de cette manière: il ne répondra pas sans avoir (d'abord) é]couté et il ne parlera pas avant d'avoir compris. (C'est) avec grande pa[tience (qu')il rendra un verdict et en étudiant la justice (qu')il en découvrira les conséquences. L'hom[me humble et d'esprit contrit ne revien-] dra [pas] en arrière jusqu'à [...L'homme fiable ne se détournera pas des voies de la justice.] Il aura [à cœur ... ses os et ses mains. (C'est) par la justice (qu')il sera racheté et par l'intelli] gence de tous [...]. (4Q421 frag. 1a ii et frag. $1 \mathrm{~b} \mathrm{10}$; complété d'après 4Q420 frag. $1 \mathrm{a}$ ii et frag. $1 \mathrm{~b}$ )

\subsection{Recueils poétiques ou liturgiques}

Recueil de bénédictions

Annexé à la Règle de la communauté, le Recueil de bénédictions (1QSb) consiste en une série de bénédictions pour les membres de la communauté 
et ses responsables. Le maśkîl y apparaît trois fois, dans les trois seuls titres préservés, comme celui qui doit prononcer les bénédictions pour les fidèles (I 1), les fils de Sadoq (III 22) et le Prince de la congrégation (V 20). La formule est toujours la même: "(Paroles de bénédiction) pour le maśkîl, pour bénir N....». La caractérisation des personnes ou des groupes et le détail des bénédictions qui leurs sont adressées supposent une connaissance adéquate aussi bien du statut de chacun et de sa place dans le plan divin.

Ainsi, les fidèles sont "ceux qui craignent [Dieu, ceux qui accomplissent] sa volonté et gardent ses commandements, ... marchent de façon parfaite [en toutes] ses [voies de] vérité, et qu'il a élus pour une alliance éternelle» (I 1-3a). Le maśkîl leur souhaite, sur un ton personnalisé, que Dieu «ouvre pour toi du (haut) des cieux, la source éternelle qui ne [déçoit] pas » et «[t'enseigne ?] dans la Congrégation des saints» (I 3b-5). Dans la bénédiction des fils de Sadoq, "les prêtres que Dieu a choisis pour affermir son alliance à [jamais]» (III 22-23) il leur demande que Dieu fasse d'eux "un ornement magnifique au milieu des saints» et qu'il renouvelle pour eux «l'alliance du sacerdoce éternel»(III 25-26). Quant au «Prince de la Congrégation", un chef militaire eschatologique appelé à rétablir «à jamais le royaume de son peuple» et à faire régner la justice dans le pays (V 21-22), il est demandé à Dieu de l'élever "vers une hauteur éternelle ", de lui accorder "[un esprit de conseil] et de puissance éternelle, un esprit de connaissance et de crainte de Dieu » et de le rendre fort "par son saint nom » (V 23-28).

\section{Hymnes}

Le terme maśkîl est attesté ou reconstruit avec vraisemblance dans l'introduction de quatre prières du rouleau des Hymnes (1QH ${ }^{a} \mathrm{~V} 12$; VII 21 ; XX 7; par. 4Q427 frag. 8 ii 10; 4Q428 frag. 12 ii 3; $1 \mathrm{QH}^{\mathrm{a}}$; XXV 34).

La première occurrence apparaît au début d'une longue prière $\left(1 \mathrm{QH}^{\mathrm{a}}\right.$ V 12 - VI 33), dans un titre assez développé et partiellement reconstruit qui évoque celui de l'Instruction sur les deux esprits: «[Un psaume pour le ma]śkîl pour qu'il se prosterne de[vant Dieu ...] les œuvres de Dieu [...] que les (personnes) simples comprennent [...] à jamais [...] et que l'humanité comprenne ce qui concerne [le ...] de chair et le conseil des esprits [d'après lequels (?)] ils se conduisent» (V 12-14). Le psaume évoque tour à tour le mystère du plan que Dieu, par son intelligence (śèkèl), a élaboré et mis en œuvre dans la création (V 17.25-30), l'opposition entre les voies de la vérité et les œuvres du mal, la faiblesse de l'être humain qui doit sa 
justification à la seule grâce de Dieu et au don de son esprit saint (V 30-35; VI 23-27). Le maśkîl insiste à plusieurs reprises sur la révélation et le don de l'esprit dont lui et ses pairs bénéficient et qui leur permettent de connaître ce mystérieux plan divin et de s'y conformer (V 19-20.35-40; VI 13-14.19-24.28):

[Béni sois-tu] Seigneur, qui place l'intelligence dans le cœur de ton serviteur pour qu'il saisisse toutes ces choses, com[prenne ...], se maitrise devant les actes impies, bénisse tous ceux qui choisissent de faire ta volonté, [choisisse tout ce] que tu aimes et abhorre tout ce que tu [hais] (...) Moi, je sais, par l'intelligence (qui vient) de toi, que selon ta bienveillance envers une personne, tu multi[plies sa part] à ton esprit saint, le faisant ainsi avancer davantage vers ton intelligence. Et selon sa proximité, il fera preuve de zèle contre les malfaisants et les tricheurs... (VI 19-25)

Il termine sa prière en soulignant le comportement qu'il adopte envers les hommes de son "conseil », auxquels il a été associé, aimant ou haïssant chacun selon son mérite (VI 28-33).

Dans l'introduction du deuxième hymne (VII 21 - VIII 41), le maśkîl proclame son amour pour Dieu et sa détermination à se conformer à sa volonté:

Béni [sois-tu, Dieu miséricordieux par] un chant, un psaume pour le maś[kîl ...] Et moi, je t'aime résolument. De tout mon cœur et de tout mon être, je me suis purifié de l'iniquité [et, sur] moi-[même, j’ai ju]ré de ne pas me détourner de tout ce que tu as commandé. Je tiendrai ferme [...] sans abandonner aucun de tes décrets. (VII 21-25a)

Attribuant son savoir à «l'intelligence» de Dieu, qui lui a ouvert «une source de lumière» (VII 25b; VIII 14), le psalmiste formule une sorte de confession de foi, ponctuée à plusieurs reprises de la formule déclaratoire «Et moi, je sais que...» (VII 25b.26.35.38-39; VIII 22.28-29.34). Il affirme sa conviction que le juste et l'impie sont créés par Dieu, l'un pour le salut et la paix éternelle, l'autre pour la colère et le châtiment: la voie de tout vivant est entre les mains de Dieu et l'homme n'y peut rien changer (VII 25b-39). Heureusement, cet humble serviteur de Dieu bénéficie de la compassion divine; appartenant au camp des justes, il proclame son désir d'être totalement purifié par l'esprit de sainteté et invité à s'approcher de Dieu pour se tenir en sa présence (VIII 22-36).

Le titre "pour le maśkîll» est reconstruit en $1 \mathrm{QH}^{\mathrm{a}} \mathrm{XX} 7 \mathrm{a}$ d'après les passages parallèles des manuscrits de la grotte 4 (4Q427 frag. 8 ii 10; 4Q428 frag. 12 ii 3). Rappelant les titres des psaumes bibliques «d'instruc- 
tion ", il introduit un long hymne (XX 7 à XXII 42) présenté comme "[une ac]tion de grâce et une prière pour tomber (en prostration) et supplier constamment, en tout temps ". Les premières lignes énumèrent des temps de prières réglés sur l'alternance de la lumière et des ténèbres, établie par le «Dieu des connaissances» et créateur de tout ce qui est et sera (XX $7 b-14 a)$.

Le psalmiste affirme ensuite: "Et moi, maśkîl, je te connais, mon Dieu, par l'esprit que tu as mis en moi et j'ai fidèlement écouté ton merveilleux conseil secret. Par ton esprit saint, tu m'as [ou]vert intérieurement à la connaissance par le mystère de ta sagesse... » (XX 14b-16a). Dans un passage partiellement mutilé, il évoque à la fin de l'oppression et l'avènement d'une période de réjouissance pour des élus qui «connaissent» Dieu et auxquels il sera donné de s'approcher de lui «selon leur discernement» pour le servir fidèlement » (XX 16b-27a). Il rappelle alors sa propre situation d'être de poussière et de cendres ne pouvant rien saisir aux actions de Dieu (XX 27a-34), sinon grâce à une révélation intérieure: "Et moi, je reste muet. Que pourrais-je dire à propos de ceci ? J'ai parlé selon ma connaissance, comme une créature pétrie d'argile. Que pourrais-je dire si tu n'ouvrais pas ma bouche? Que pourrais-je comprendre si tu ne me donnais pas le discernement? Que pourrais-je dire si tu ne (le) révélais pas à mon cœur ?...» (XX 35-37a).

Des thèmes similaires reviennent dans la colonne XXI, où il est en outre précisé que «l'armée de connaissance », c'est-à-dire la troupe céleste, contribue à « raconter à la chair des prouesses, et des décrets à celui qui est né [d'une femme]» (XXI 9-10). Grâce à cette révélation et à la compassion de Dieu qui l'a amené à lui dans une relation d'alliance, le fidèle espère échapper au jugement et à la destruction (XXI 11-30). Mais, contrairement à ceux qui ne peuvent comprendre (XXII 6-8), il devra se montrer persévérant au milieu des épreuves et ne pas se laisser détourner de l'alliance par des traîtres (XXII 9-15). Après l'avoir remercié encore une fois de lui avoir ouvert le cœur à son intelligence, il conclut en bénissant le "Dieu des connaissances » qui l'a affermi et en qui il espère avec confiance (XXII 28-38.)

Le dernier hymne mentionnant le maśkîl se trouve en 1QHa XXV 23 - XXVII 3. Il s'agit d'un assemblage de fragments, dont le texte est reconstitué à l'aide de passages parallèles de trois rouleaux des Hymnes de la grotte 4 (4Q427-428, 431). Il a aussi des affinités avec un hymne d'autoglorification préservé dans un des manuscrits de la Règle de la guerre (4Q491 frag. 11 i - 12; voir Duhaime 2004, 35-40). Il débute par «Pour 
le maśkîl, un cha[nt, un cantique pour...»] (XXV 34). Dans le haut de la col. XXVI, on reconstruit un passage évoquant l'exaltation du psalmiste au milieu des anges: "Qui est comme moi parmi les êtres divins?... Car moi, ma place est avec les êtres divins... " (XXVI 4-7; voir 4Q427 frag. 7 i 11; 4Q431 frag. 14 ). Celui-ci invite l'assemblée des «bien-aimés » à chanter la louange de Dieu dans sa sainte demeure, en compagnie de la troupe céleste (XXVI 9-14a; 4Q427 frag. 7 i 13-18a).

Dieu est béni pour avoir fait connaître sa puissance "en scellant les mystères et en révélant des choses cachées, en redres[sant ceux qui trébuchent $(. .$.$) et en remettant sur pied ceux qui espèrent la connaissance] »$ (XXVI 14b-15; voir 4Q427 frag. 7 i 18-20). Il juge les impies avec colère et traite les fidèles avec compassion, fait disparaître l'injustice et apporte la paix et la joie, élevant le pauvre sur une hauteur éternelle, en compagnie des êtres divins (XXVI 17-30; voir 4Q427 frag. 7 i 21- ii 11). Une autre partie de la bénédiction prend la forme d'une confession de foi de la part des élus qui bénéficient d'une relation privilégiée à Dieu:

[Nous te connaissons,] Dieu de justice, et nous comprenons [ta] vé[rité, roi de gloire, car nous voyons ton zèle] dans (ta) forte puissance. [Nous] reconnaissons [tes jugements dans l'abondance ta miséricorde et dans tes merveilleux pardons]. Qu'est la chair par rapport à cela ? Et que [valent la poussière et la cendre pour pouvoir raconter ces choses continuellement], prendre place [devant toi et faire communauté avec les fils du ciel ?]. (XXVI 32-36; voir 4Q427 frag. 7 ii $14-18$ )

\section{Chants pour l'holocauste du sabbat}

Le terme maśkîl apparaît dans les "Chants pour l'holocauste du sabbat ", un recueil de textes cultuels, peut-être d'origine pré-essénienne, prévus pour accompagner l'offrande de chacun des treize premiers sabbats de l'année, ou éventuellement s'y substituer (Newsom 2000, 887-889). Il est préservé dans les titres de six chants et figurait sans doute dans les autres (4Q400 frag. 3 ii et 58 ; 4Q401 frag. 1-2 1; 4Q403 frag. 1 i 30; ii 18; 4Q405 frag. 20 ii-22 6 et parallèle en 11Q17 VII 9; 4Q406 frag. 14$)^{10}$. Ces titres sont tous formulés de la même manière: "Pour le maśkîl, chant pour l'holocauste du (numéro) sabbat, le (date) du mois». Ils sont suivis d'un appel à une catégorie d'anges à louer Dieu:

10. On peut le reconstruire à quelques endroits, y compris dans un exemplaire fragmentaire découvert à Massada (4Q400 frag. 1 i 1; 4Q405 frag. 8-9 1; Mas 1k I 8). 
Pour le maśkîl, chant pour l'holocauste du septième sabbat, le seize du mois. Célébrez le Dieu des lieux élevés! (Ô vous) les exaltés parmi tous les anges (doués) de connaissance. Que les saints de Dieu exaltent le roi de gloire qui sanctifie par sa sainteté tous ses saints. (Ô vous) chefs des louanges de tous les dieux, louez le Dieu des louanges, (le) majestueux. (4Q403 frag. $1 \mathrm{i}$ 30-32a)

Les cinq premiers chants exposent l'établissement du sacerdoce céleste, les tâches assignées aux anges et la louange qu'ils offrent à Dieu. Les trois chants suivants portent principalement sur la louange offerte par les "princes en chef» (les archanges) et leurs seconds, au cœur du temple du ciel, et culminent dans la description du trône divin et des grands prêtres angéliques. Les cinq derniers décrivent le temple céleste en progressant de l'extérieur jusqu'au trône, les louanges des anges qui y vont et viennent, et les sacrifices offerts par les prêtres angéliques. Le maśkîl qui proclame ces chants est ainsi associé étroitement à la liturgie du temple divin, au milieu des anges.

Les thèmes de la connaissance et du discernement sont assez fréquents dans ces textes. Dans le chant pour l'offrande du premier sabbat, «ceux qui s'approchent de la connaissance (divine) » sont probablement les anges, établis comme "ministres de la Présence " dans le sanctuaire céleste (4Q400 frag. 14-6; voir 4Q401 frag. 17 4). Dans le chant du second sabbat, les archanges sont appelés «dieux de la connaissance »; ils proclament la majesté de la royauté divine "selon leur connaissance» et les prêtres humains, avec leur "langue de poussière", s'associent à eux pour exalter le "Dieu de la connaissance ", dont l'intelligence les surpasse tous" (4Q400 frag. 2 1-10; voir 4Q401 frag. 14 i 7-8). Des archanges semblables sont qualifiés ailleurs de "princes" ayant accès aux "mystères" divins et transmettant «des choses cachées ", un savoir «sorti des lèvres du Roi », à des anges de rang inférieur ou à un groupe privilégié d'êtres humains (4Q401 frag. 14 ii 2-8). Le chant pour le cinquième sabbat fait allusion à l'implication des anges dans la guerre eschatologique qui précèdera un renouvellement du monde en conformité avec un plan dont Dieu seul connaît les détails:

Car du Dieu de la connaissance sont issus tous [les êtres, à jamais. Et de sa connaissance et de son dessein est survenu tout ce qui a été décrété depuis tou]jours. Ayant fait les pre[miè]res (choses) [en leur temps, (il fera) les suivantes en leur moment. De ceux qui ont la connaissance, nul ne] peut comprendre [ses révélations merveilleuses] avant qu'[il] n'a[gisse. Et quand il agit, (même) les dieux ne peuvent discerner ce qu'il a en vue. Car] eux- 
mêmes [font partie de ses œuvres glorieuses:] avant même d'exister, ils faisaient partie de son plan. (4Q402 frag. 4 12-15; restauré d'après Mas frag. $1 \mathrm{k}$ I 2-7)

Dans le chant pour le sixième sabbat, des anges de haut rang ( «des chefs exaltés») sont appelés à bénir tour à tour les anges et peut-être les humains "qui ont la connaissance des choses éternelles", "les exaltés de la connaissance", "tous les di[eux qui s'appro]chent de la connaissance de sa (divine) vérité ", " tous ceux qui ont un puissant discernement ", "tous ceux qui affermissent la connaissance » (4Q403 frag 1 11-24; par. 4Q404 frag. $21-6$; 4Q405 frag. 3 ii 1-16). Des expressions similaires reviennent abondamment dans le chant pour le septième sabbat qui invite les «exaltés parmi tous les dieux de connaissance» à louer Dieu (4Q403 frag. $1 \mathrm{i}$ 30-31). Les motifs de louange sont que Dieu fait exister tous les êtres par sa parole et sa connaissance et «il juge, par sa puissance, tous les esprits d'intelligence». Aussi «tous les dieux de connaissance» qui confessent la gloire de Dieu et «tous les esprits de justice» qui confessent sa vérité "accordent leur connaissance en fonction des jugements de sa bouche» (i 38-39). Le chant pour le huitième sabbat rapporte les psaumes et bénédictions prononcés dans le temple céleste par sept «princes en second ... qui ont la connaissance des (choses) éternelles»(4Q403 frag. 1 ii 18; voir 4Q405 frag. 8-9 3; frag. 13 5). On y fait référence à «sept mystères de connaissance dans le mystère merveilleux des sept «enceintes [les plus] sacré[es]» (4Q403 frag. 1 ii 27), à ceux qui «font luire la connaissance parmi les dieux de lumière» (ii 35), etc.

Un fragment contenant probablement des vestiges du chant du dixième sabbat mentionne la présence ou l'action d' "esprits de connaissance et de compréhension» (4Q405 frag. 17 3). Un autre, provenant du chant du onzième sabbat, décrit des éléments architecturaux du temple céleste comme l'œuvre d' "esprits de connaissance, de vérité et de justice dans le saint [des saints]» (4Q405 frag. 19 4; voir 11Q17 VI 5). Le chant du douzième sabbat nous fait pénétrer dans "le taberna[cle du Dieu de] connaissance", devant lequel les chérubim se prosternent avant de se redresser pour le bénir (4Q405 frag. 20 ii-21-22 8). Vers la fin de ce chant, il est aussi question de "dieux de connaissance » et "de saints anges» circulant à travers les portails sacrés (4Q405 frag 23 i 8). Le dernier chant, celui du treizième sabbat, dit à propos des «chefs de l'exaltation (divine) » qu'il y a en eux «des langues de connaissance» et qu' "ils bénissent le Dieu de la connaissance et toutes ses œuvres glorieuses ", célébrant «sa connaissance profonde (ou «intelligente») et son discernement glorieux» (4Q405 frag. 23 ii 12-13). 
Chants pour le maśkîl

La figure du maśkîl revient à deux reprises dans un recueil d'hymnes à son usage préservé en deux exemplaires (4Q510 frag. 14; 4Q511 frag. 2 i 1 ; voir Baillet 1982, 215-262). Ces textes ont pour but de chasser les démons et les esprits mauvais en évoquant, sous forme d'incantations, la puissance et la gloire de Dieu:

[...] louanges. Béné[dictions (adressées) au r]oi de gloire. Paroles d'actions de grâces en psaumes de [...] au Dieu des connaissances, splendeur des $\mathrm{pu}$ [issan]ces, Dieu des êtres divins, Seigneur de tous les saints. [Son] empi[re] (s'étend) sur tous les puissants de force, et devant la force de sa puissan[ce], tous sont pris de panique et se dispersent, et ils prennent la fuite devant la majesté de la dem[eure de] gloire de sa royauté. Et moi, je suis un maśkîl, qui proclame la majesté de sa splendeur, pour effrayer et ter[rifier] tous les esprits d'anges de corruption et les esprits de bâtards: démons, Lilith, hiboux et [chats sauvages ...] et ceux qui frappent à l'improviste pour l'égarement de l'esprit d'intelligence. (4Q510 frag. 1 1-6)

La seconde mention se trouve dans le titre d'un chant du deuxième exemplaire, dont le contenu évoque la fin de la domination du mal et le début d'une ère de bonheur: "Pour le maśkîl, [...] chant [...Louez le nom de] sa sainteté et exaltez-le, vous tous qui connaissez [...] Et le chef des dominations, il l'a arrêté sans [...joie per]pétuelle et vie éternelle, pour faire briller une lumière [...]» (4Q511 frag. 2 1-4). On suggère également de reconstruire le terme maśkîl au début du deuxième chant de ce manuscrit: "[Pour le maśkîll]. Deuxième [ch]ant, pour effrayer ceux qui inspirent la crainte [...]» (4Q511 frag. 8 4). Dans un des fragments subséquents, le psalmiste proteste de la droiture de ses propos et l'attribue à la connaissance qu'il a reçue de Dieu:

[Y a-t-il des insanités] dans mes paroles? Il n'y en a pas. Dans ce qui sort de mes lèvres? Pas de chose inutile. [...] et mon esprit d'intelligence et $[. .$. œuvre d'impiété. Car Dieu me scrute, et toutes les œuvres d'impiété je (les) hais. Car Dieu a fait luire dans mon cœur la connaissance de l'intelligence... (4Q511 frag. 18 ii 5-8)

Un peu plus loin il rend également grâce à Dieu de l'avoir doté de connaissance malgré la fragilité de son être: «Et moi [je] te [rends grâce] car, pour ta gloire, tu as mis la connaissance dans l'intimité de ma poussière, pour [te] lou[er], alors que moi, (c'est) d'une mixture (que) j'ai été] constitué [et d'argile (que)] j'ai été pétri. (4Q511 frag. 28-29 2-4; voir frag. $48-48$ et 511 ). 
Dans un autre passage, enfin, il affirme avoir reçu la capacité de saisir la motivation profonde des actions humaines, ce qui l'habilite à porter un jugement sur le juste et l'impie:

Quant à moi, ma langue acclamera ta justice, car tu l'as déliée. Sur mes lèvres, tu as placé une source de louange et dans mon cœur le secret du principe de toutes les actions de l'homme et des intentions des œuvres des parfaits de conduite et des jugements sur tout service qu'ils accomplissent, pour justifier (le) juste en ta vérité et condamner (l')impie en sa culpabilité, pour proclamer «Paix à tous les hommes de l'alliance» et dé[cla]rer d'une voix terrible: "Malheur à tous ceux qui la rompent». (4Q511 frag. 63 iii 1-5)

\subsection{Quelle forme de discernement?}

Dans l'ensemble de ces textes, le maśkîl apparaît comme une figure exemplaire de connaissance, de discernement et de leadership dont l'autorité s'exerce dans plusieurs domaines de la vie communautaire. Dans le Document de Damas et la Règle de la communauté, il se présente comme une personne possédant une connaissance à transmettre concernant la nature et la place des humains dans la création, la diversité des esprits qui les influencent et la rétribution eschatologique qui leur est réservée selon leur conduite (1QS III 13 - IV 26). Son savoir porte aussi sur l'interprétation de la Torah, au sujet de laquelle il est également un guide pour sa communauté (4Q256 IX 1; 4Q258 I 1). Sa connaissance permet au maśkîl de reconnaître les «hommes de la fosse" pour s'en écarter, de jauger chaque membre de sa communauté, de lui assigner le rang qui lui convient et de l'aider à progresser dans l'accomplissement de la volonté de Dieu (1QS IX 12-26). Enfin, grâce aux «secrets merveilleux» que Dieu a mis en son cœur, il peut saisir l'ensemble du plan créateur, des origines à «ce qui sera éternellement », ce qui l'élève pratiquement au rang des anges (1QS XI 3-8).

Les écrits de sagesse vont dans le même sens. Dans l'Instruction, la figure du maśkîl n'apparaît qu'une fois, au pluriel et dans un sens générique, pour désigner diverses personnes susceptibles de faire pénétrer plus avant le disciple dans le mystère de l'existence et l'aider à améliorer sa conduite (4Q417 frag. 1 i 6-13; 4Q 418 frag. 81-81a 17). Ailleurs, le maśkîl est non seulement l'initiateur ou l'instructeur des novices de divers niveaux, mais aussi celui qui accompagne et corrige ses compagnons au fil des jours (4Q298; 4Q420-421).

Les textes poétiques et liturgiques nous font assister à un déploiement encore plus complet de la connaissance du maśkîl, principalement en ce qui 
concerne le plan de Dieu sur le monde et sur l'humanité, mais aussi les réalités célestes dans toute leur splendeur. Dans ces textes, le maśkîl évoque sa propre fragilité, celle des humains en général et leur totale dépendance de Dieu; mais, en contrepartie, il rend grâce pour sa purification et celle des autres élus (1QH V 12 - VI 33; VII 21 - VIII 41; XX 7 - XXII 42; XXV 23 - XXVII 3). Ses bénédictions aux fidèles, au prêtre ou au prince de la congrégation, suggèrent qu'il connaît à la fois le rôle dévolu à chacun des serviteurs de Dieu et l'avenir radieux qui lui est réservé (1QSb). La révélation des secrets divins, de la gloire et de la puissance de Dieu lui permet d'affronter sans crainte les forces démoniaques (4Q510-511) et de partager dès maintenant la compagnie des anges dans le temple céleste en s'associant mystiquement au culte qui s’y déroule (4Q400-407; 11Q17; Mas 1k).

En somme, le maśkîl apparaît comme celui qui, par grâce divine, a appris à voir le monde du point de vue de Dieu et s'est investi totalement dans un rôle d'élu cheminant vers l'exaltation ultime. Cette revendication n'est jamais justifiée explicitement: énoncée de diverses manières par le maśkîl, elle se propose comme telle à la reconnaissance de ses pairs et semble contenir sa propre légitimation. On peut cependant penser que dans un groupe qui se livrait intensément à l'étude et à la délibération en commun, comme en témoigne la Règle de la communauté (voir 1QS VI 6-10), le savoir et la compétence du maśkîl n'était pas exclusivement le fruit d'une illumination personnelle, mais aussi de recherche, de méditation, de discussion et de validation avec d'autres membres (voir Newsom 1990b, 375).

Il est impossible sur la seule base des textes qui en font mention, de préciser le statut ou l'identité concrète du maśkîl ou des maśkîlîm dont ils parlent. Certains traits, par exemple le fait qu'il soit chargé des bénédictions et son intérêt pour le temple céleste, suggèrent qu'il pourrait s'agir d'un prêtre; d'autres évoquent plutôt un déplacement de la figure du sage vers l'apocalyptique ou la mystique, un peu comme dans le livre de Daniel. On a aussi suggéré de comprendre le terme maśkîl, surtout lorsqu'il apparaît dans des titres, comme une expression abstraite, "pour l'instruction ", plutôt qu'une référence à une personne concrète (Hawley 2006); mais cette approche n'a guère eu d'échos. D'autres chercheurs distinguent divers aspects de la figure du maśkîl et les associent à des niveaux de tradition ou des milieux d'origine distincts, selon qu'il s'agit de textes pré-sectaires ou sectaires, ou encore de textes provenant vraisemblablement de groupes apparentés à un même mouvement mais vivant dans des conditions ou à des époques variées (Elgvin 2013; Hempel 2006; Lange 2008). 


\subsection{Quelle contribution à la construction identitaire?}

Quoi qu'il en soit, d'un point de vue synchronique et sociologique, le terme maśkîl semble plutôt suggérer le type idéal du membre et du guide accompli à l'intérieur d'un mouvement apocalyptique. Il joue un rôle d'évaluation des aptitudes des candidats et préside à leur initiation dans la communauté, instruit ses pairs et les aide à progresser dans leur compréhension du plan de Dieu et leur adhésion à une vie pure et sainte, partage son cheminement spirituel et son expérience mystique dans la prière, par laquelle il contribue aussi à la protection contre les forces du mal et appelle la bénédiction de Dieu sur ses serviteurs. On peut penser que cette figure du maśkîl s'incarnait concrètement dans un ou plusieurs membres, parmi les anciens, peut-être, dont les pairs reconnaissaient à la fois l'humilité et les dons exceptionnels, qui avaient développé une fine connaissance de l'âme humaine, parfaitement intégré la tradition interprétative, la vision apocalyptique, l'eschatologie et la mystique du groupe et qui, pour ces raisons, représentaient pour ses membres les modèles les plus attrayants et les guides les plus sûrs. Ils contribuaient ainsi étroitement à modeler l'identité de leur groupe.

Cet aspect a été approfondi par quelques auteurs. Carol Newsom (1990a) a suggéré que, sur le plan rhétorique, la Règle de la communauté utilise la figure du maśkîl pour faire parcourir à son lecteur les diverses étapes de la vie d'un membre du groupe sectaire: admission, initiation, instruction, vie en communauté, leadership. Le personnage ainsi construit, explique-t-elle ailleurs, incarne de manière particulièrement prononcée les valeurs de la secte:

His ability to make subtle distinctions, informed by a knowledge of mysteries and a sense of eschatological destiny, his reserve with outsiders, amounting even to a dissembled self, creates a sense of mysterious power that constitutes part of his appeal both to outsiders and insiders. His disciplined orientation to God's will is shaped by practices of nearly continuous temporally structured worship, and his sense of self is formed through the cultivation of a radical alterity between divine and human nature and gratitude for being saved from human sinfulness and its consequences. (Newsom 2004, 173)

Les écrits de sagesse, qu'ils soient de provenance sectaire ou non, contribuent à faire accepter l'autorité de ceux qui connaissent «le mystère de l'être» et à asseoir la conduite des membres sur une vision du monde qui lui donne une légitimation inscrite dans un ordre immuable. Ainsi, les 
éditeurs des Paroles du maśkîl aux fils de l'aurore interprètent l'insistance de ce document sur l'ordre cosmique, comme un moyen de démontrer aux novices que Dieu a établi chaque être selon sa mesure propre et que chacun doit occuper la place qui lui a été assignée, dans l'univers aussi bien que dans la communauté (Pfann et Kister 1997, 18).

En évoquant fréquemment la communion avec les anges, les Hymnes attribuent un statut à part aux membres de la communauté de salut et leur font miroiter le sort ultime auquel ils sont promis. Les Chants pour l'holocauste du sabbat vont encore plus loin. Ils en procurent une expérience "virtuelle" en coordonnant la prière des humains et celle des troupes célestes grâce à l'observance du même calendrier cultuel et à la contemplation du temple d'en-haut. Ce faisant, ils compensent pour la rupture que ce groupe (dont plusieurs membres proviennent de la classe sacerdotale) a effectuée avec le temple terrestre contrôlé par des prêtres illégitimes:

The vivid description of the angelic priests and the heavenly temple, amounting to a sense of virtual presence in the celestial sanctuary, would have served to reaffirm the Qumran priests' sense of their own identity and validity despite their separation from the Jerusalem temple. The experience of the Sabbath Songs was perhaps a foretaste of the common priestly service with the angels, which figures prominently in the maśkîl's blessing of priests in 1QSb. (Newsom 1990b, 380-381)

De même, Joseph L. Angel estime que les Chants du sage (4Q510-511) étaient récités en présence de la communauté et éventuellement par elle, ce qui amenait ses membres à se percevoir comme réalisant ses idéaux essentiels de pureté parfaite, de connaissance d'ordre surnaturel, et à se sentir impliqués dans une bataille cosmique, en compagnie des anges:

(...) the Maskil's claims about his own impressive qualities and values represent much more than a confirmation of his privileged standing "to frighten" the demons. His speech also served to transmit vital knowledge about ritual participants to ritual participants. As the embodiment of key Qumranite ideals, the Maskil served as a template with which worshipers were to identify. No matter what the mechanics of the ritual performance, its public repetition would have solidified acceptance of the symbolic world and web of relationships assumed by the text. Participants were thus transformed from ordinary recluses to central figures in the cosmic battle between the forces of good and evil. More importantly, in identifying with the Maskil, they came to share in his Godgranted gifts and to understand that in this epic battle they were currently safe from the threat of demonic attack. That it is not necessary to posit that the Maskil was actually present during the 
performance of the ritual. The formulaic and idealized quality of his words are more readily understood as prototypical than as distinctively personal expressions, and as such, they offered themselves for easy adoption by worshipers. (Angel 2012, 26)

Quelle que soit l'origine de ces textes en rapport avec le maśkîl, dans la mesure où ils faisaient partie de la "bibliothèque " de Qumrân, ils pouvaient être utilisés par les membres de la communauté qui y vivait ou par les membres de groupes apparentés. Ils contribuaient ainsi puissamment, en évoquant cette figure exemplaire de discernement, à structurer et à renforcer leur identité autour d'une figure type qui en incarnait le savoir, la vision du monde, les vertus et les espoirs.

\section{Conclusion}

Il n'existe pas dans la bibliothèque de Qumrân de terme spécifique ou de traité systématique et complet pour parler du discernement spirituel. Le discernement s'incarne plutôt dans des figures emblématiques, celles du Maître de justice d'autrefois et celle du maśkîl contemporain inscrit dans son sillage, et dans les textes qui, en véhiculant leur portrait, leur enseignement et leur expérience spirituelle, les proposent comme des maîtres à suivre et des modèles à imiter. Dans les deux cas, il s'agit d'élus qui ont reçu de Dieu une connaissance de "ses mystères merveilleux " et une capacité de décoder sa volonté qui transcende le savoir humain commun et s'apparente à celui des anges. Reconnus comme tels par ceux qui adhèrent à leur vision du monde, assimilent leurs leçons et marchent sur leurs traces, ils deviennent un modèle et une référence pour les membres d'une communauté que leur image sécurise et dont ils représentent l'idéal de connaissance et de vie pure et sainte susceptible de conduire à l'exaltation et au salut. Ils jouent de cette manière un rôle déterminant dans la construction et le renforcement de l'identité du groupe, contribuent à légitimer l'autorité de ses dirigeants, à favoriser l'acceptation de l'ascèse et la discipline qui s'y pratiquent, à faire expérimenter dès maintenant quelque chose du bonheur ultime réservé à ceux qui tiennent ferme dans une voie socialement marginale, mais présentée comme la voie véritable. 


\section{Références}

Abegg, M. G., Bowley, J. E. et al. (2003), The Dead Sea Scrolls Concordance, vol. 1. The Non-Biblical Scrolls from Qumran, Leiden / Boston, Brill.

Angel, J. L. (2012), "Maskil, Community, and Religious Experience in the Songs of the Sage (4Q510-511) ", Dead Sea Discoveries, 19, p. 1-27.

Baillet, M. (1982), Qumrân Grotte 4 III (4Q482-4Q520), Oxford, Clarendon (DJD VII).

BerTHELOT, K. (2013), "À propos de quelques œuvres de la Loi (4QMMT) », dans K. Berthelot, T. Legrand et M. LANGlois, dir., La bibliothèque de Qumrân, vol. 3a. Deutéronome et Pentateuque dans son ensemble, Paris, Cerf, p. 647-687.

Brooke, G. J. (2005), "The "Apocalyptic" Community. The Matrix of the Teacher of Righteousness ", dans L. M. Mills et B. G. WRIGHT, III, dir., Conflicted Boundaries in Wisdom and Apocalypticism, Atlanta, Society of Biblical Literature, p. 37-53.

Cothenet, É. (1963), «Le Document de Damas», dans J. Carmignac, É. Cothenet et H. Lignée, dir., Les textes de Qumran. Traduits et annotés, vol. 2., Paris, Letouzey et Ané, p. 129-204.

DJD (1955-2010), Discoveries in the Judaean Desert, vol. I-XL Oxford, Clarendon.

Duhaime, J. (2004), The War Texts: 1QM and related manuscripts, New York/London, T \& T Clark.

Duhaime, J. et Legrand, T. (2010), Les Rouleaux de la mer Morte: textes de Qoumrân, Paris, Cerf.

Elgvin, T. (1997), "4Q420. Ways of Righteousness; 4Q421. Ways of Righteousness", dans T. Elgvin et al., Qumran Cave 4 xv. Sapiential Texts, Part 1, Oxford, Clarendon (DJD XX), p. 173-202. (2013), "משכיל ", dans H. J. FABry, dir., Theologisches Wörterbuch zu den Qumrantexten. Band 2, Stuttgart, Kohlhammer, col. 802-806.

Finsterbusch, K. (2013), "ירה ", dans H. J. Fabry, dir., Theologisches Wörterbuch zu den Qumrantexten. Band 2, Stuttgart, Kohlhammer, col. 269-275.

Guilbert, P. (1961), "La règle de la communauté", dans J. CARMignaC et P. Guilbert, dir., Les textes de Qumran. Traduits et annotés, vol. 1, Paris, Letouzey et Ané, p. 9-80. 
Harkins, A. K. (2012), "Who is the Teacher of the Teacher Hymns? Re-examining the Teacher Hymns Hypothesis Fifty Years Later ", dans E. F. Mason, S. I. Thoas, A. Schfield et E. Ulrich, dir., A Teacher for all Generations. Essays in Honor of James C. VanderKam, Leiden/ Boston, Brill, p. 449-467.

(2013), "Thanksgiving Hymns», dans L. H. Feldman, J. L. Kugel et H. Lawrence, Ancient Jewish writings related to Scripture, Philadelphia, Jewish Publication Society, p. 2018-2094.

Hawley, R. (2006), "On Maskil in the Judean Desert Texts ", Henoch, 28, p. 43-77.

Hempel, C. (1998), The Laws of the Damascus Document: Sources, Tradition, and Redaction, Boston, Brill (Studies on the texts of the desert of Judah).

(2006), «Maskil (im) and rabbim: from Daniel to Qumran », dans C. Hempel et J. Lieu, dir., Biblical traditions in transmission, Leiden / Boston, Brill, p. 133-156.

Jeremias, G. (1963), Der Lehrer der Gerechtigkeit, Göttingen, Vandenhoeck und Ruprecht.

Jokiranta, J. (2006), "The Prototypical Teacher in the Qumran Pesharim. A Social-Identity Approach ", dans P. F. EsLer, dir., Ancient Israel: The Old Testament in Its Social Contexts, Minneapolis, Fortress, p. 254-263.

(2008), "Social Identity Approach. Identity-Construction Elements in the Psalms Pesher ", dans F. García Martínez et M. Popovíc, dir., Defining identities: we, you, and the other in the Dead Sea Scrolls: proceedings of the fifth meeting of the IOQS in Gröningen, Leiden/Boston, Brill, p. 85-109.

Kampen, J. (2011), Wisdom literature, Grand Rapids, Eerdmans.

Koenen, K. (1993), "שכל ", dans H. J. Fabry et H. Ringreen, dir., Theologisches Wörterbuch zum Alten Testament. Band 7, Stuttgart, Kohlhammer, col. 781-795.

Knibi, M. A. (2000), "Teacher of Righteousness", dans L. H. Schiffman et J. C. VanderKam, dir., Encyclopedia of the Dead Sea scrolls, vol. 2, New York/Toronto, Oxford University Press, p. 918-921.

LANGe, A. (2008), "Sages and Scribes in the Qumran Literature", dans L. G. Perdue, dir., Scribes, sages, and seers. The sage in the Eastern Mediterranean world, Göttingen, Vandenhoeck \& Ruprecht (Forschungen zur Religion und Literatur des Alten und Neuen Testaments), p. 271-293. 
Laperrousaz, E.-M. (1997), "Le Maître de justice ", dans E.-M. Laperrousaz, dir., Qoumrân et les Manuscrits de la mer Morte: un cinquantenaire, Paris, Cerf, p. 391-408.

Newsom, C. A. (1990a), "Apocalyptic and the Discourse of the Qumran Community ", Journal of Near Eastern Studies, 49, p. 135-144.

(1990b), "The Sage in the Literature of Qumran: The Functions of the Maśkîl», dans J. G. Gammie et L. G. Perdue, dir., The Sage in Israel and the ancient Near East, Winona Lake, Eisenbrauns, p. 373-382.

(2000), "Songs of the Sabbath Sacrifice», dans L. H. Schiffman et J. C. VanderKam, dir., Encyclopedia of the Dead Sea scrolls, New York/Toronto, Oxford University Press, p. 887-889.

(2004), The self as symbolic space: constructing identity and community at Qumran, Leiden/Boston, Brill.

Parry, D. W. et Tov, E. (2004), The Dead Sea Scrolls Reader, Leiden, Brill.

Pfann, S. J. et Kister, M. (1997), "4QcryptA Words of the Maskil to All Sons of Dawn", dans T. Elgvin et al., Qumran Cave 4 xv. Sapiential Texts, Part 1, Clarendon (DJD XX), p. 1-30.

Qimron, E., Strugnell, J. et al. (1994), Qumran Cave 4 V. miqtsat ma'ase ha-torah, Oxford, Clarendons (DJD X).

Rey, J.-S. (2009), 4QInstruction: Sagesse et eschatologie, Leiden, Brill.

Stegemann, H., Schuller, E. M. et al. (2009), Qumran cave 1. 3, 1 QHodayot $^{a}$ : with incorporation of 1 QHodayot $t^{b}$ and 4QHodayot ${ }^{a-f}$, Oxford, Clarendon (DJD XL).

Strugnell, J., Harrington, D. J. et al. (1999), Qumran Cave 4 xxiv. Sapiential Texts, Part 2. 4QInstruction (Mûsar Le Mevin): 4Q415 ff., Oxford, Clarendon (DJD XXXIV).

Stuckenbruck, L. T. (2010), "The Legacy of the Teacher of Righteousness in the Dead Sea Scrolls", dans E. G. Chazon, B. H. Amaru et R. Clements, dir., New Perspectives on Old Texts: Proceedings of the Tenth International Symposium of the Orion Center for the Study of the Dead Sea Scrolls and Associated Literature. 9-11 January, 2005, Leiden/ Boston, Leiden, Brill, p. 23-49.

Tov, E. (2006), dir., The Dead Sea Scrolls Electronic Library, Leiden, Brill. 
Ulfgard, H. (1998), "The Teacher of Righteousness, the History of the Qumran Community, and our Understanding of the Jesus Movement: Texts, Theories and Trajectories ", dans F. H. Cryer et T. L. Thompson, dir., Qumran between the Old and New Testaments, Sheffield, Sheffield Academic Press, p. 310-346.

\section{Résumé}

Les manuscrits de la mer Morte témoignent d'une communauté qui affirme connaître le «véritable » plan de Dieu sur le monde et y répondre de manière appropriée en pratiquant un style de vie rigoureux, sous la conduite de maîtres éclairés. Pour soutenir cette prétention, elle s'appuie sur deux figures principales de discernement, le "maître de justice" (moreh tsèdeq), dont l'enseignement a joué un rôle déterminant dans la structuration de la communauté, et le "sage» ou l'«instructeur» (maśkîll), chargé de montrer la voie aux recrues et de faire progresser les membres. Dans cet article, chacune de ces figures-types est d'abord présentée à partir des textes qui la mentionnent, après quoi on identifie la forme que prend le discernement qui lui est attribué et comment cette représentation contribue à la construction et au renforcement de l'identité sociale du groupe.

\section{Abstract}

The Dead Sea Scrolls mirror a community which claims to know the "true» plan that God has set for his creation and to correspond to it by their rigorous lifestyle, under the guidance of enlightened masters. To support this claim, they draw on two key figures of discernment, the Teacher of righteousness (moreh tsedeq), whose teaching has been critical for the structuration of the community, and the "wise one" or the "Instructor" (maśkîl), the one who was showing the way to the novices and helping the members to make progress. In this paper, each of these typical figures is first presented through the texts in which they are mentioned; then is identified which form took the discernment attributed to those figures and how these representations contributed to the construction and consolidation of the group's social identity. 